\title{
Bounded error identification of systems with time-varying parameters
}

\author{
J.M. Bravo, T. Alamo and E.F. Camacho
}

\begin{abstract}
This paper presents a new approach to guaranteed system identification for time-varying parameterized discrete-time systems. A bounded description of noise in the measurement is considered. The main result is an algorithm to compute a set that contains the parameters consistent with the measured output and the given bound of the noise. This set is represented by a zonotope, that is, an affine map of a unitary hypercube. A recursive procedure minimizes the size of the zonotope with each noise corrupted measurement. The zonotope allows us to take into account the time-varying nature of the parameters in a non conservative way. An example has been provided to clarify the algorithm.
\end{abstract}

\section{INTRODUCTION}

Research activity in system identification has been strong in recent years. New identification technics are needed for advance robust and adaptive control. Different identification schemes can be considered depending on the way the uncertainty is described. The stochastic approach [15], [25] assumes a probabilistic description of the uncertainty. However, in many situations, it is difficult to obtain the statistical properties of the noise. An interesting alternative is to consider a bounded but unknown uncertainty. Setmembership identification approach assumes a parametric model and an additive bounded error. These methods compute a feasible solution set (FSS) of parameters. This feasible set is compatible with the model structure, obtained measurements and the considered uncertainty. Setmembership approach can be used to identify systems with parametric and nonparametric uncertainty.

When a linear model is considered, the FSS obtained by the set-membership approach is a polytope and it is possible to compute it exactly. An algorithm that provides the facets of the solution set is presented in [6]. Alternatively, the FSS is represented by its vertices in [18]. In [20], a polyhedric cone is used to represent the FSS. This representation can be used when the initial set of parameters is not bounded.

The main drawback of the exact methods is the associated computational burden and the complexity of the representation of the exact feasible solution set. In order to reduce these limitations, approximated feasible solution sets (AFSS), that bound the corresponding FSS, can be used. Boxes, ellipsoidss, paralellotopes and limited-complexity polyhedrons are used to represent the AFSS.

T. Alamo and E.F. Camacho are with the Departamento de Ingeniería de Sistemas y Automática, Universidad de Sevilla, Escuela Superior de Ingenieros, Camino de los Descubrimientos s/n. 41092 Sevilla, SPAIN \{alamo, eduardo\}@cartuja.us.es

J.M. Bravo is with the Departamento de Ingeniería Electrónica, Sistemas Informáticos y Automática, Universidad de Huelva, Carretera Huelva - La Rábida 21071 Palos de la Fra. Huelva, Spain caro@uhu.es
A sequence of ellipsoids [10] can be used to represent the AFSS. Each sample time, the new measurement is used to obtain a minimal size ellipse that bounds the FSS. Tangent hyperplanes can be used [3] to obtain better results. Other related results can be found in [2], [9]. The ellipsoidal method can be used with non linear models too. For example, in [12], the nonlinear dependence with respect to the parameters of the model is avoided using an equivalent representation in a higher dimensional space.

Polyhedral AFSS have also been used in error-bounded identification. A minimal box is used in [16] and [11] to represent the AFSS. If the model is polynomial in the parameters of the system, signomial programming can be used to find a box that bounds the FSS [17]. Interval branch and bound algorithms are used in [13] to describe the AFSS as a union of boxes.

In [24], an algorithm to compute the minimal volume parallelotope that bounds the intersection of a previous parallelotope with a strip is presented. This strip bounds the parameters that are consistent with the measurement. A recursive limited-complexity polyhedric method to bound the AFSS is used by [21]. A box is used by [4] to compute the recursion.

In order to estimate the parameters of linear time-varying systems with bounded error, a modification of the algorithm proposed in [4] is presented in [5]. A form of data forgetting is incorporated to ensure that old data which are no representative of the current system are discarded and not allowed to polarize the parameter estimates. Two recursive polyhedric description algorithms are presented in [22] for time-varying systems. In the first algorithm, at each step $k$, the polyhedron is expanded to account for possible parameter variations. In the second algorithm the Chebyshev center of the polyhedron is moved to the median hyperplane of the region consistent with the new data.

In this paper, a new bounded-error identification scheme for time-varying parameter systems is proposed. The approach relies on the use of zonotopes to represent the approximated feasible set solution (AFSS). A zonotope is an affine map of a unitary hypercube. Boxes and parallelotopes are particular cases of zonotopes. The main contribution of this paper is an algorithm that bounds the feasible solution set by means of a zonotope. The size of the zonotope is reduced at each sample time using the new measurement. Moreover, we show how the time-varying nature of the parameters can be taken into account by means of the zonotope in a direct and non-conservative way.

The paper is organized as follows. In section II the 
problem is formulated. The bounded-error identification algorithm is presented in section III. Section IV reports one example. The paper draws to a close with a section of conclusions.

\section{PROBLEM FORMULATION}

A quite general problem appearing in many scientific and technical fields is to make some inference on a dynamic system based on some general information on it and on a given sequence of noise corrupted measurements. In particular, in the context of set membership identification, a bound of the vector of parameters $\theta \in \mathbb{R}^{n}$, that characterizes the dynamics of the system, is obtained from the given measurements. We will assume that each measurement is a function of a regression vector, the parameter vector $\theta$ and a given bounded error:

Assumption 1: A set of measurements $y_{1}, y_{2}, \ldots, y_{N}$ is provided. Each measurement $y_{k} \in \mathbb{R}$ is related with the parameter vector $\theta_{k}$ and the regression vector $r_{k}$ by means of the following expression:

$$
y_{k}=r_{k}^{\top} \theta_{k}+e_{k}
$$

where $e_{k}$ represents the considered error. This error belongs to a bounded set: $e_{k} \in E=\{e \in \mathbb{R}:|e| \leq \sigma\}$.

A multivariable measurements approach is equivalent to obtain several measurements in the same sample time $k$, so a generalization to multivariable measurements is straightforward.

The term $e_{k}$ bounds the effect of non modelled dynamics, perturbations to the system, noise in the measurements, etc. To obtain a tighter bound of $e_{k}$, it is possible to include in $\theta_{k}$ an additive perturbation to the system. The example provided in this paper shows this possibility.

Assumption 2: The set of parameters to be estimated is time-varying. A bounded expansion is considered, that is: $\left\|\theta_{k}-\theta_{k-1}\right\|_{\infty} \leq \gamma$ for all $k$. The scalar $\gamma>0$ is the expansion factor considered.

Given a set of measurements, the feasible solution is defined as the set of parameters that are consistent with the measurements, the given bounds of the error and the parameter expansion. More precisely:

Definition 1 (Feasible Solution Set): Let us suppose that the pairs $\left(y_{k}, r_{k}\right), k=1, \ldots, N$ are given. We say that the vector of parameters $\theta$ belongs to the Feasible Solution Set if there exists $\theta_{1}, \theta_{2}, \ldots, \theta_{N}$ such that:

(i) $\theta=\theta_{N}$

(ii) $\left|y_{k}-r_{k}^{\top} \theta_{k}\right| \leq \sigma, k=1, \ldots, N$

(iii) $\left\|\theta_{k}-\theta_{k-1}\right\|_{\infty} \leq \gamma, k=2, \ldots, N$

It is a difficult task to compute the FSS. If the complexity of the representation has to be kept simple, an outer bound of the feasible solution set can be used.

Definition 2 (Approximated Feasible Solution Set): An Approximated Feasible Solution Set, denoted AFSS, is a set that fulfils $F S S \subseteq A F S S$.

In this paper we propose the use of zonotopes to obtain approximated feasible solution sets. The main advantage of this choice is that the time-varying nature of the uncertainty is easily represented by this type of sets. In the next section a new set membership identification algorithm is presented.

\section{BOUNDED-ERROR SYSTEM IDENTIFICATION} METHOD

Let us suppose that the parameters of system (1) do not change with respect to time (they are time-invariant). Then, given the pair $d_{k}=\left(y_{k}, r_{k}\right)$ at instant $k$, there is a strip $F_{d_{k}}=\left\{\theta \mid-\sigma \leq r_{k}^{\top} \theta-y_{k} \leq \sigma\right\}$ that is consistent with $d_{k}$. The FSS can be computed exactly by the recursion $F S S_{k+1}=F S S_{k} \cap F_{d_{k}}$.

In order to consider the time-varying case, it is important to recall the notion of Minkowski sum, unitary interval and unitary box:

Definition 3 (Minkowski sum): The Minkowski sum of two sets $X$ and $Y$ is defined by $X \oplus Y=\{x+y$ : $x \in X, y \in Y\}$.

Definition 4 (Unitary interval): The unitary interval is $\mathbf{B}=[-1,1]$.

Definition 5 (Unitary box): A unitary box, denoted by $\mathbf{B}^{m}$, is a vector compound by $m$ unitary intervals.

Note that the bound on the rate of change of the parameters: $\left\|\theta_{k+1}-\theta_{k}\right\|_{\infty} \leq \gamma$ can be interpreted in terms of the Minkowski sum:

$$
\theta_{k+1}=\theta_{k} \oplus \gamma \mathbf{B}^{n}
$$

where $n$ is the dimension of the parameter vector. Therefore, in case of time-varying parameters, the $F S S$ can be obtained by means of the following recursion:

$$
F S S_{k+1}=\left(F S S_{k} \cap F_{d_{k}}\right) \oplus \gamma \mathbf{B}^{n} .
$$

In this paper, a new identification method that uses zonotopes to obtain an outer bound of $\left(F S S_{k} \cap F_{d_{k}}\right) \oplus \gamma \mathbf{B}^{n}$ is proposed.

Definition 6 (Zonotope of order $m$ ): Given vector $p \in$ $\mathbb{R}^{n}$ and matrix $H \in \mathbb{R}^{n \times m}$, the set $p \oplus H \mathbf{B}^{m}$ is denominated a zonotope of order $m$. Note that this is the Minkowski sum of the segments defined by the columns of matrix $H$.

The order $m$ is a measure for the geometrical complexity of the zonotopes. The main advantage of zonotopes in the context of time-varying parameters is that the Minkowski sum of a zonotope and a box is a zonotope. That is, if the intersection $F S S_{k} \cap F_{d_{k}}$ is approximated by means of a zonotope $p \oplus H \mathbf{B}^{m}$ then

$$
F S S_{k+1} \subseteq p \oplus H \mathbf{B}^{m} \oplus \gamma \mathbf{B}^{n}=p \oplus\left[\begin{array}{ll}
H & \gamma I
\end{array}\right] \mathbf{B}^{m+n}
$$

With this approach, the order of the zonotope is increased at each sample time. Therefore, it is desirable to use an algorithm to bound a high order zonotope by a lower order one. A simple algorithm to reduce the complexity of a given zonotope can be found, for example, in [14] and [8].

Note that if the approximation of $F S S_{k}$, denoted $A F S S_{k}$, is represented by a zonotope, then a procedure 
to bound the intersection of a zonotope and a strip is required in order to obtain $A F S S_{k} \cap F_{d_{k}}$. In the following property, a parameterized family of zonotopes containing the intersection between a zonotope and a strip is given.

Property 1: Given the zonotope $\mathcal{X}=p \oplus H \mathbf{B}^{r} \subset \mathbb{R}^{n}$, the strip $\mathcal{S}=\left\{x \in \mathbb{R}^{n}:\left|c^{\top} x-d\right| \leq \sigma\right\}$ and the vector $\lambda$, define:

- $\hat{p}(\lambda)=p+\lambda\left(d-c^{\top} p\right)$

- $\hat{H}(\lambda)=\left[\begin{array}{ll}\left(I-\lambda c^{\top}\right) H & \sigma \lambda\end{array}\right]$

Then, $\mathcal{X} \cap \mathcal{S} \subseteq \hat{\mathcal{X}}(\lambda)=\hat{p}(\lambda) \oplus \hat{H}(\lambda) \mathbf{B}^{r+1}$

PRoOF: Let us suppose that $x \in \mathcal{X} \cap \mathcal{S}$. Then $x \in \mathcal{X}=$ $p \oplus H \mathbf{B}^{r}$. This implies that there is $z \in \mathbf{B}^{r}$ such that:

$$
x=p+H z
$$

In fact, adding and substracting $\lambda c^{\top} H z$ to previous equality:

$$
x=p+\lambda c^{\top} H z+\left(\mathrm{I}-\lambda c^{\top}\right) H z
$$

From $x \in \mathcal{X} \cap \mathcal{S}$ it is inferred that $x \in \mathcal{S}=\left\{x \in \mathbb{R}^{n}\right.$ : $\left.\left|c^{\top} x-d\right| \leq \sigma\right\}$. Thus, there exists $w \in[-1,1]=\mathbf{B}^{1}$ such that $c^{\top} x-d=\sigma w$. Taking into account equation (2) it results that $c^{\top}(p+H z)-d=\sigma w$. That is:

$$
c^{\top} H z=d-c^{\top} p+\sigma w
$$

Substituting this equality in equation (3), the following is obtained:

$$
\begin{gathered}
x=p+\lambda\left(d-c^{\top} p+\sigma w\right)+\left(I-\lambda c^{\top}\right) H z= \\
p+\lambda\left(d-c^{\top} p\right)+\lambda \sigma w+\left(I-\lambda c^{\top}\right) H z= \\
\hat{p}(\lambda)+\left[\left(I-\lambda c^{\top}\right) H \quad \sigma \lambda\right]\left[\begin{array}{c}
z \\
w
\end{array}\right]= \\
\hat{p}(\lambda)+\hat{H}(\lambda)\left[\begin{array}{c}
z \\
w
\end{array}\right] \subseteq \hat{p}(\lambda) \oplus \hat{H}(\lambda) \mathbf{B}^{r+1}=\hat{\mathcal{X}}(\lambda)
\end{gathered}
$$

The parametric zonotope $\hat{\mathcal{X}}(\lambda)$ bounds the intersection, but it is interesting to find an optimal parameter $\lambda^{*}$ that provides a minimal size zonotope.

\section{A. Minimizing the volume of the intersection}

Let us suppose that we want to minimize the volume of $\hat{\mathcal{X}}(\lambda)$. In this case, we should choose $\lambda$ in such a way that the volume of the zonotope $\hat{\mathcal{X}}(\lambda)=\hat{p}(\lambda) \oplus \hat{H}(\lambda) \mathbf{B}^{r+1}$ is minimized. It is well known (see [19], [23]) that the volume of a zonotope $a \oplus D \mathbf{B}^{m} \subset \mathbb{R}^{n}$ is given by:

$\operatorname{Vol}\left(a \oplus D \mathbf{B}^{m}\right)=\sum_{i=1}^{N(n, m)} 2^{n}\left|\operatorname{det}\left[\begin{array}{lll}D_{s_{1}(i)} & \ldots & D_{s_{n}(i)}\end{array}\right]\right|$

where $N(n, m)$ denotes the number of different ways of choosing n elements from a set of m. $D_{i}$ denotes the $i$-th column of $D$. Integers $s_{j}(i), j=1, \ldots, n, i=1, \ldots, N$ denote each one of the different ways of choosing $n$ elements from a set of $\mathrm{m}$. That is, these integers satisfy:

$$
1 \leq s_{1}(i)<s_{2}(i)<\ldots<s_{n}(i) \leq m
$$

Moreover, if $i \neq j$ then:

$$
\left[s_{1}(i) \quad \ldots \quad s_{n}(i)\right] \neq\left[\begin{array}{lll}
s_{1}(j) & \ldots & s_{n}(j)
\end{array}\right]
$$

Theorem 1: Consider the zonotope $\hat{\mathcal{X}}(\lambda)=p+\lambda(d-$ $\left.c^{\top} p\right)+\left[\left(I-\lambda c^{\top}\right) H \quad \sigma \lambda\right]$ defined in property 1 . The volume of $\hat{\mathcal{X}}(\lambda)$ is given by the following expression:

$$
\begin{gathered}
\operatorname{Vol}\left(\hat{p}(\lambda) \oplus \hat{H}(\lambda) \mathbf{B}^{r+1}\right)= \\
\sum_{i=1}^{N(n, r)} 2^{n}\left|1-c^{\top} \lambda\right|\left|\operatorname{det}\left(A_{i}\right)\right|+ \\
\sum_{i=1}^{N(n-1, r)} \sigma 2^{n}\left|\operatorname{det}\left[\begin{array}{ll}
B_{i} & v_{i}
\end{array}\right]\right|\left|v_{i}^{\top} \lambda\right|
\end{gathered}
$$

where $A_{i}$ denotes each of the different matrices that can be obtained choosing $n$ columns from matrix $H$. On the other hand, $B_{i}$ denotes each of the different matrices that can be obtained choosing $n-1$ columns from $H$ and $v_{i}$ is orthonormal to $\operatorname{Imag}\left(B_{i}\right)$.

The proof of the previous result has been provided by the authors in [1] (in the context of nonlinear state stimation). For the sake of completeness, the proof of the result is also included in this paper.

PROOF:

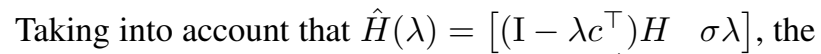
expression corresponding to the volume of $\hat{\mathcal{X}}(\lambda)$ is:

$$
\begin{gathered}
\operatorname{Vol}\left(\hat{p}(\lambda) \oplus \hat{H}(\lambda) \mathbf{B}^{r+1}\right)= \\
\sum_{i=1}^{N(n, r)} 2^{n}\left|\operatorname{det}\left[\left(\mathrm{I}-\lambda c^{\top}\right) A_{i}\right]\right|+ \\
\sum_{i=1}^{N(n-1, r)} 2^{n}\left|\operatorname{det}\left[\left(\mathrm{I}-\lambda c^{\top}\right) B_{i} \quad \sigma \lambda\right]\right|
\end{gathered}
$$

Where $A_{i}$ denotes each of the different matrices that can be obtained choosing $n$ columns from matrix $H$. On the other hand, $B_{i}$ denotes each of the different matrices that can be obtained choosing $n-1$ columns from $H$. Let us recall the following well known properties of the determinant of a matrix:

- $\operatorname{det}(A B)=\operatorname{det}(A) \operatorname{det}(B)$.

- Given vectors $a, b \in \mathbb{R}^{n}: \operatorname{det}\left(\mathrm{I}+a b^{\top}\right)=1+b^{\top} a$.

The above equalities will be used to calculate the different terms that appear in the expresion of the volume of $\hat{\mathcal{X}}(\lambda)$. We will distinguish between two different classes of terms:

- Terms of the form $\operatorname{det}\left[\left(\mathrm{I}-\lambda c^{\top}\right) A_{i}\right]$ : In this case,

$$
\operatorname{det}\left[\left(\mathrm{I}-\lambda c^{\top}\right) A_{i}\right]=
$$

$\operatorname{det}\left(\mathrm{I}-\lambda c^{\top}\right) \operatorname{det}\left(A_{i}\right)=\left(1-c^{\top} \lambda\right) \operatorname{det}\left(A_{i}\right)$

- Terms of the form $\operatorname{det}\left[\begin{array}{ll}\left(\mathrm{I}-\lambda c^{\top}\right) B_{i} & \sigma \lambda\end{array}\right]$ : Note that $B_{i}-\lambda c^{\top} B_{i}$ is obtained substracting from each column of $B_{i}$ the last column of 
$\left[\left(\mathrm{I}-\lambda c^{\top}\right) B_{i} \sigma \lambda\right]$ multiplied by a scalar. It is well known that the determinant of a matrix does not change if a column is added or substracted from another one. This implies that:

$$
\operatorname{det}\left[\left(\mathrm{I}-\lambda c^{\top}\right) B_{i} \quad \sigma \lambda\right]=\operatorname{det}\left[\begin{array}{ll}
B_{i} & \sigma \lambda
\end{array}\right]
$$

Two different cases must be distinguished: $\operatorname{rank}\left\{B_{i}\right\}<n-1$ and $\operatorname{rank}\left\{B_{i}\right\}=n-1$. If rank $\left\{B_{i}\right\}<n-1$ then $\operatorname{det}\left[\begin{array}{ll}B_{i} & \sigma \lambda\end{array}\right]=0$. In the following, it will be supposed that $\operatorname{rank}\left\{B_{i}\right\}=n-1$. It is clear, under this assumption, that there exists $v_{i}$ such that $v_{i}^{\top} v_{i}=1$ and $v_{i}^{\top} * B_{i}=0$. That is, $v_{i}$ is orthonormal to $\operatorname{Imag}\left(B_{i}\right)$. Therefore, $\Phi_{i}=\left[\begin{array}{ll}B_{i} & v_{i}\end{array}\right]$ is not singular. Note that:

$$
\begin{gathered}
\operatorname{det}\left[\begin{array}{ll}
B_{i} & \sigma \lambda
\end{array}\right]=\operatorname{det}\left[\begin{array}{lll}
B_{i} & \left(v_{i}-v_{i}+\sigma \lambda\right)
\end{array}\right]= \\
\operatorname{det}\left(\Phi_{i}+\left(\sigma \lambda-v_{i}\right)\left[\begin{array}{llll}
0 & 0 & \ldots & 1
\end{array}\right]\right)= \\
\operatorname{det}\left(\Phi_{i}\right) \operatorname{det}\left(I+\Phi_{i}^{-1}\left(\sigma \lambda-v_{i}\right)\left[\begin{array}{llll}
0 & 0 & \ldots & 1
\end{array}\right]\right)= \\
\operatorname{det}\left(\Phi_{i}\right)\left(1+\left[\begin{array}{llll}
0 & 0 & \ldots & 1
\end{array}\right] \Phi_{i}^{-1}\left(\sigma \lambda-v_{i}\right)\right)
\end{gathered}
$$

Taking into account that $\left[\begin{array}{llll}0 & 0 & \ldots & 1\end{array}\right] \Phi_{i}^{-1}=v_{i}^{\top}$ :

$$
\begin{gathered}
\operatorname{det}\left(\Phi_{i}\right)\left(1+v_{i}^{\top}\left(\sigma \lambda-v_{i}\right)\right)= \\
\operatorname{det}\left(\Phi_{i}\right)\left(1+\sigma v_{i}^{\top} \lambda-1\right)=\sigma \operatorname{det}\left(\Phi_{i}\right)\left(v_{i}^{\top} \lambda\right)
\end{gathered}
$$

To conclude, the volume of $\hat{\mathcal{X}}(\lambda)$ is given by the following expression:

$$
\begin{gathered}
\operatorname{Vol}\left(\hat{p}(\lambda) \oplus \hat{H}(\lambda) \mathbf{B}^{r+1}\right)= \\
\sum_{i=1}^{N(n, r)} 2^{n}\left|1-c^{\top} \lambda\right|\left|\operatorname{det}\left(A_{i}\right)\right|+ \\
\sum_{i=1}^{N(n-1, r)} \sigma 2^{n}\left|\operatorname{det}\left[\begin{array}{ll}
B_{i} & v_{i}
\end{array}\right]\right|\left|v_{i}^{\top} \lambda\right|
\end{gathered}
$$

Note that $\operatorname{Vol}\left(\hat{p}(\lambda) \oplus \hat{H}(\lambda) \mathbf{B}^{r+1}\right)$ is a convex function of $\lambda$. This means that obtaining the vector $\lambda$ that minimizes the volume of the zonotope is a convex problem. Therefore, specialized algorithms can be used.

\section{B. Tight strip}

To obtain better performance, the proposed identification algorithm must use tight strips. Next, the notion of tight strip is recalled.

Definition 7 (Support hyperplanes): We say that the hyperplanes $S_{1}=\left\{x: c^{\top} x=r\right\}$ and $S_{2}=\left\{x: c^{\top} x=q\right\}$ are tangent to the zonotope $Z=p \oplus H \mathbf{B}^{m}$ if $r$ and $q$ satisfy:

$$
r=\max _{x \in Z} c^{\top} x \text { and } q=\min _{x \in Z} c^{T} x
$$

Note that given a zonotope $p \oplus \stackrel{x \in Z}{H \mathbf{B}^{m}}$ and the vector $c \in \mathbb{R}^{n}$, the scalars $r$ and $q$ that characterize the support hyperplanes can be obtained easily:

$$
r=c^{\top} p+\left\|H^{\top} c\right\|_{1}, \quad \text { and } \quad q=c^{\top} p-\left\|H^{\top} c\right\|_{1} .
$$

Definition 8 (Support strip): Given zonotope $Z=p \oplus$ $H \mathbf{B}^{m}$ and vector $c$, the support strip is defined by $F_{S}=$ $\left\{x: q \leq c^{\top} x \leq r\right\}$, where $q$ and $r$ characterize the support hyperplanes.

Definition 9 (Tight strip): Given zonotope $Z=p \oplus$ $H \mathbf{B}^{m}$ and a strip $F=\left\{x: q_{0} \leq c^{T} x \leq r_{0}\right\}$, the tight strip is obtained by $F_{T}=F_{S} \cap F$, where $F_{S}$ is the support strip defined by $c$ and $Z$.

With these definitions: $F \cap Z=F_{S} \cap F \cap Z=F_{T} \cap Z$. Taken into account that $F_{T} \subseteq F$, it results that better identification results are obtained if we intersect the zonotope with the tighter strip $F_{T}$.

\section{Identification algorithm}

This section presents a new recursive algorithm to identify bounded error linear systems with time-varying parameters. The algorithm is based on the results presented in the previous sections. Let us suppose that the zonotope $A F S S_{k}=\mathcal{X}=p \oplus H \mathbf{B}^{r}$ bounds the feasible set solution at sample instant $k$. Then:

1) Obtain input-output data and build a strip that bounds the consistent parameters $F_{d_{k}}=\left\{\theta \mid-\sigma \leq r_{k}^{\top} \theta-\right.$ $\left.y_{k} \leq \sigma\right\}$.

2) Use the zonotope $A F S S_{k}$ and the strip $F_{d_{k}}$ to build the tight strip $F_{T_{k}}$.

3) Build the intersection of the zonotope $A F S S_{k}$ and the tight strip $F_{T_{k}}$. The resulting parametric zonotope is $\hat{\mathcal{X}}(\lambda)=\hat{p}(\lambda) \oplus \hat{H}(\lambda) \mathbf{B}^{r+1}$.

4) Compute $\lambda^{*}$ in order to minimize the size of the parametric zonotope $\hat{\mathcal{X}}(\lambda)$.

5) Expand the obtained set $\hat{\mathcal{X}}\left(\lambda^{*}\right)$ in order to take into account the possible change in the parameters, that is: $A F S S_{k+1}=\hat{\mathcal{X}}\left(\lambda^{*}\right) \oplus \gamma I \mathbf{B}^{n}=\hat{p}\left(\lambda^{*}\right) \oplus$ $\left[\hat{H}\left(\lambda^{*}\right) I \gamma\right] \mathbf{B}^{r+1+n}$, where $I \in \mathbb{R}^{n \times n}$ is the identity matrix.

With this proposal, at each sample time, the order of the zonotope is increased. Therefore, it is desirable to use an algorithm to bound a high order zonotope by a lower order one. This algorithm can be found in [14] or [8].

\section{EXAMPLE}

The new identification method is used to identify the linear system with time-varying parameters:

$$
y_{k}=0.9 y_{k-1}-0.8 y_{k-2}-u_{k}-0.8 u_{k-1}+w_{k}+e_{k}
$$

where $y_{k}$ is the output, $u_{k}$ the input, $w_{k}$ is a perturbation to the system and $e_{k}$ an error term. So, the vectors of the system (1) are defined by:

$r_{k}=\left[\begin{array}{c}y_{k-1} \\ y_{k-2} \\ u_{k} \\ u_{k-1} \\ 1\end{array}\right] \quad \theta_{c}=\left[\begin{array}{c}0.9 \\ -0.8 \\ -1 \\ -0.8 \\ 0\end{array}\right] \quad \sigma=0.05 \gamma=\left[\begin{array}{l}0.01 \\ 0.01 \\ 0.01 \\ 0.01 \\ 0.05\end{array}\right]$

The fifth component of $\theta$ is the perturbation $w_{k}$ with an expansion factor defined by $\left|w_{k}-w_{k+1}\right| \leq 0.05$. A 
random bounded input $\left|u_{k}\right|<5$ is considered to obtain 100 measurements. The initial conditions are $y_{-2}=y_{-1}=$ $u_{-1}=0$. The initial search space is:

$$
C S F_{0}=\theta_{c}+\left[\begin{array}{ccccc}
2 & 0 & 0 & 0 & 0 \\
0 & 2 & 0 & 0 & 0 \\
0 & 0 & 2 & 0 & 0 \\
0 & 0 & 0 & 2 & 0 \\
0 & 0 & 0 & 0 & 2
\end{array}\right] \mathbf{B}^{5}
$$

The order of the zonotopes has been limited to forty segments. The figure 1 shows the volume evolution of $A F S S_{k}$. The figures $2,3,4$ y 5 present the guaranteed bounds of the parameters. The figure 6 shows the obtained guaranteed bound of the perturbation of the system. A comparison with the parallelotope method [24] has been made. To include the variation of the parameters, the size of the parallelotope is increased with the algorithm presented in [7]. The bounds obtained by the parallelotope method are worse than the bounds obtained by the proposed method.

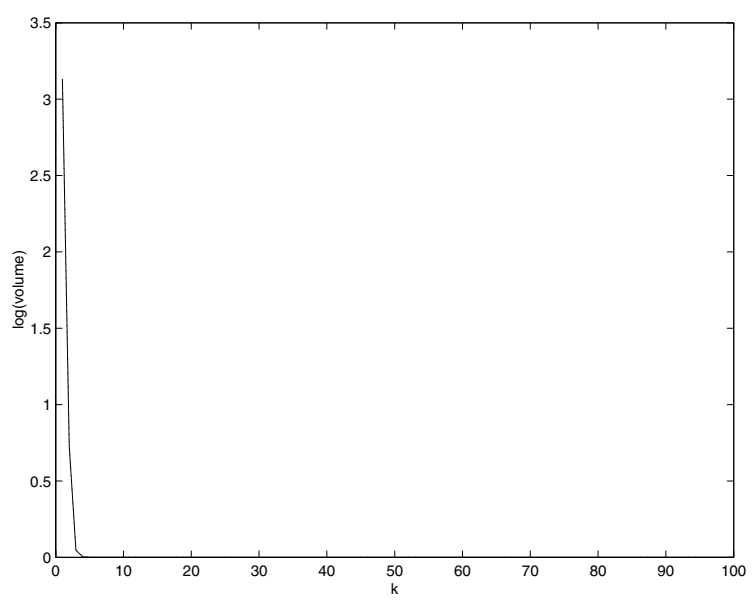

Fig. 1. Volume evolution.

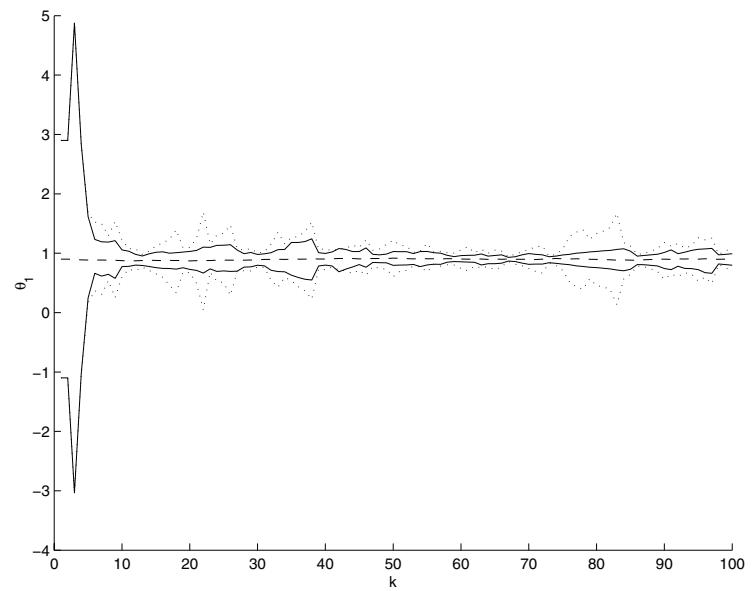

Fig. 2. Dashed line: Evolution of $\theta_{1}$. Continuous line: Guaranteed bound provided by the new identifi cation method. Dotted line: Guaranteed bound provided by the parallelotope method.

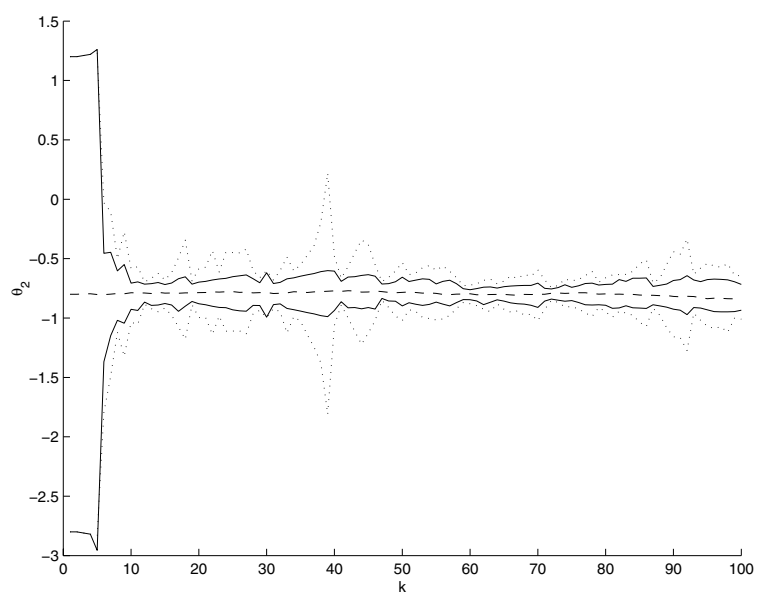

Fig. 3. Dashed line: Evolution of $\theta_{2}$. Continuous line: Guaranteed bound provided by the new identifi cation method. Dotted line: Guaranteed bound provided by the parallelotope method.

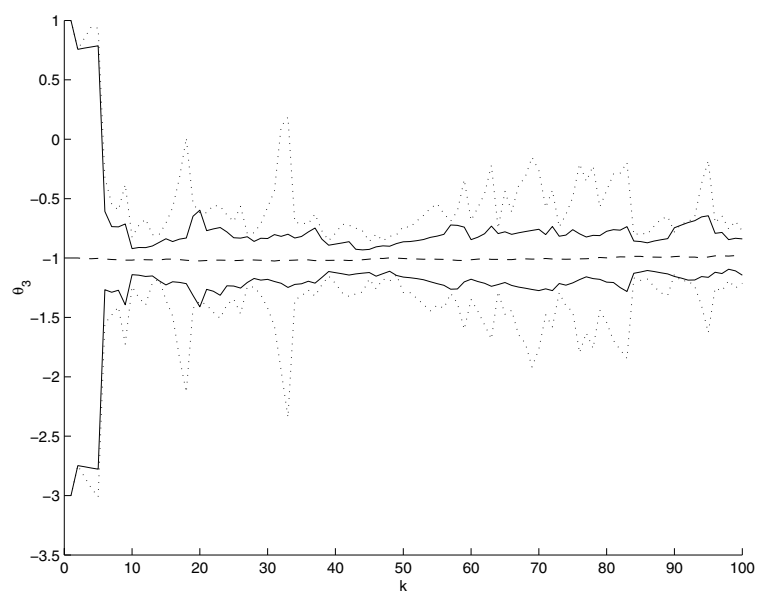

Fig. 4. Dashed line: Evolution of $\theta_{3}$. Continuous line: Guaranteed bound provided by the new identifi cation method. Dotted line: Guaranteed bound provided by the parallelotope method.

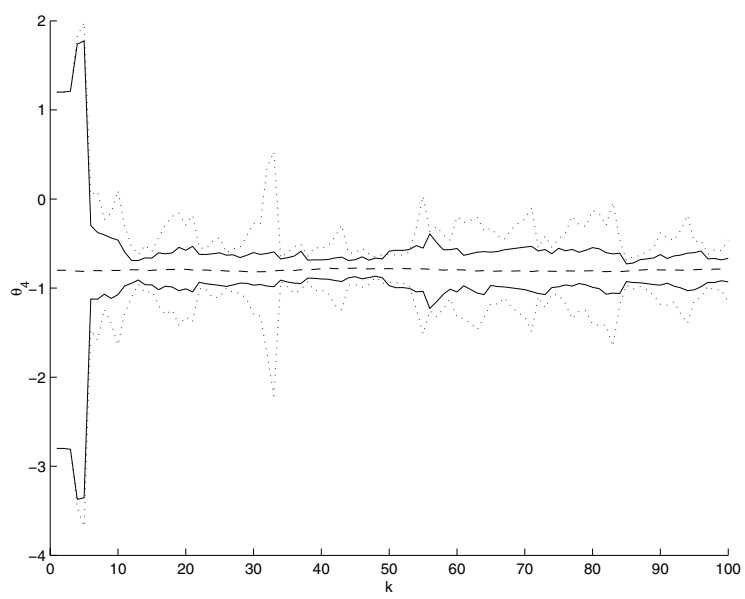

Fig. 5. Dashed line: Evolution of $\theta_{4}$. Continuous line: Guaranteed bound provided by the new identifi cation method. Dotted line: Guaranteed bound provided by the parallelotope method. 


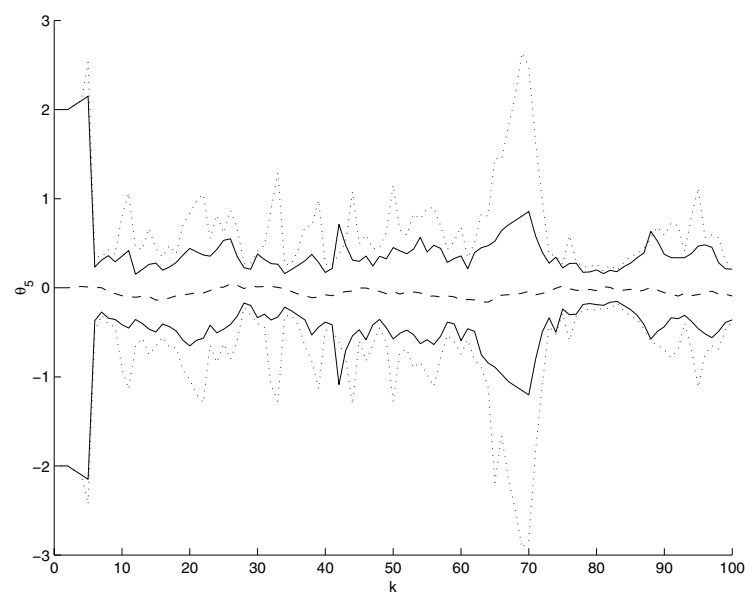

Fig. 6. Dashed line: Evolution of $\theta_{5}$. Continuous line: Guaranteed bound provided by the new identifi cation method. Dotted line: Guaranteed bound provided by the parallelotope method.

\section{CONCLUSIONS}

A new bounded-error identification method for system with time-varying parameters has been proposed. The method computes the set of parameters that are consistent with the model, the bounded noise and the obtained measurements. The proposed approach can be applied to linear models with time-varying parameters. The approximated feasible set of parameters is represented by zonotopes. New operations with zonotopes and strips have been proposed. Each new measurement is used to further reduce the size of the zonotope that bounds the feasible set of parameters. The properties of the zonotopes allows us to take into account the time-varying nature of the parameters in a nonconservative way. An illustrative examples is given.

\section{REFERENCES}

[1] T. Alamo, J.M. Bravo, and E.F. Camacho. Guaranteed state estimation by zonotopes. In Proceedings 42nd IEEE Conference on Decision and Control, Hyatt Regency Maui, Hawaii,USA, December 9-12 2003.

[2] L.V.R. Arruda and G. Favier. A review and a comparison of robust estimation methods. In Prep. 9th IFAC/IFORS Symposium on Identifi cation and System Parameter Estimation, pages 1027-1032, Budapest,Hungary, 1991.

[3] G. Belforte, B. Bona, and V. Cerone. Parameter estimation algorithms for a set-membership description of uncertainty. Automatica, 26:887$898,1990$.

[4] G. Belforte and T.T. Tay. Two new algorithms for sequential parameter estimation with unknown but bounded noise. In Proc. 29th IEEE Conference on Decision and Control, volume 6, pages 3546-3551, Honolulu, USA, December 1990.

[5] G. Belforte, Y.T. Teo, and T.T. Tay. Recursive parameter estimation for time-varying systems in presence of unknown but bounded measurement noise. In Proceedings of International Conference on Intelligent Control and Instrumentation, SICICI '92., volume 2, pages 945 - 950, Singapore, February 1992.

[6] V. Broman and M.J. Shensa. A compact algorithm for the intersection and approximation of n-diemnsional polytopes. In Proc. 12th IMACS world Congr. on Scientifi c Computation, volume 2, pages 473-476, Paris, France, July 1988.

[7] L. Chisci, A. Garulli, and G. Zappa. Recursive state bounding by parallelotopes. Automatica, 32:1049-1056, 1996.
[8] C. Combastel. A state bounding observer based on zonotopes. In European Control Conference 2003, Cambridge, England, 2003.

[9] S. Dasgupta and Y.F. Huang. Asymptotically convergent midifi ed recursive least-squares with data-dependent updating and forgetting factor for systems with bounded noise. IEEE Transactions on Infromation Theroy, 33(3):383-392, 1987.

[10] E. Fogel and Y.F. Huang. On the value of information in system identifi cation - bounded noise case. Automatica, 18:229-238, 1982.

[11] J.L. Goffi $\mathrm{n}$ and J.P. Vial. Cutting planes and column generation techniques with the projective algorithm. J. of Optim. Theory and Appl, 65:409-429, 1990.

[12] U.D. Hanebeck. Recursive nonlinear set-theoretic estimation based on pseudo ellipsoids. In Proc. International Conference on Multisensor Fusion and Integration for Intelligent Systems, pages 159-164, Paris, France, Aug. 2001.

[13] L. Jaulin, M. Kieffer, O. Didrit, and E. Walter. Applied Interval Analysis with Examples in Parameter and State Estimation, Robust Control and Robotics. Springer-Verlag, 2001.

[14] W. Kühn. Rigorous computed orbits of dynamical systems without the wrapping effect. Computing, 61(1):47-67, 1998.

[15] L. Ljung. System Identifi cation. Prentice Hall, Upper Saddle River, NJ, 1999.

[16] M. Milanese and G. Belforte. Estimation theory and uncertainty intervals evaluation in presence of unknown but bounded error: linear families of models and estimators. IEEE Transactions on Automatic Control, 27:408-414, 1982.

[17] M. Milanese and A. Vicino. Estimation theory for nonlinar models and set membership uncertainty. Automatica, 27:403-408, 1991.

[18] S.H. Mo and J.P. Norton. Recursive parameter-bounding algorithms which compute polytope bounds. In Proc. 12th IMACS world Congr. on Scientifi c Computation, volume 2, pages 477-480, Paris, France, July 1988.

[19] H.L. Montgomery. Computing the volume of a zonotope. Amer. Math. Monthly, 96:431, 1989.

[20] H. Piet-Lahanier and E. Walter. Practical implementation of an exact and recursive algorithm for characterizing likelohoods sets. In Proc. 12th IMACS world Congr. on Scientific Computation, volume 2, pages 481-483, Paris, France, July 1988.

[21] H. Piet-Lahanier and E. Walter. Polyhedric approximation and tracking for bounded-error models. In Proc. IEEE International Symposium on Circuits and Systems, volume 1, pages 782-785, Chicago, USA, May 1993.

[22] H. Piet-Lahanier and E. Walter. Bounded-error tracking of timevarying parameters. IEEE Transactions on Automatic Control, 39:1661-1664, 1994.

[23] G.C. Shephard. Combinatorial properties of associated zonotopes. Canadian Journal of Mathematics, 26:302-321, 1974.

[24] A. Vicino and G. Zappa. Sequential aproximation of feasible parameter sets for identifi cation with set membership uncertainty. ITAC, 41(6):774-785, 1996.

[25] E. Walter and L. Pronzato. Identifi cation of Parametric Models from Experimental Data. Springer-Verlag, 1997. 